\title{
THE GREEN TEA POLYPHENOL EGCG MODULATES NGF, BDNF, AND CAMKII- $\alpha$ PATHWAYS TO ALLEVIATE NEUROLOGICAL DAMAGE IN AUTISM-INDUCED RATS
}

\author{
OGUZHAN OZDEMIR ${ }^{1,2 *}$ \\ 'Department of Crop and Animal Production, Technical Sciences Vocational School, Batman University, \\ Batman, Turkey \\ ${ }^{2}$ Central Laboratory Application and Research Center, Batman University, Batman, Turkey
}

\begin{abstract}
This study investigated molecular-level neuroprotective effects of EGCG, an important component of green tea, on rats in which an experimental autism model was created using PPA. Twenty-eight 21-d-old male Sprague-Dawley rats were randomly assigned to the following four groups ( $\mathrm{n}=7$ each): 1) control: given a placebo, 2) EGCG: given $100 \mathrm{mg} / \mathrm{kg}$ EGCG by oral gavage for $35 \mathrm{~d}$, 3) PPA: given $500 \mathrm{mg} / \mathrm{kg} \mathrm{BW} \mathrm{sc} \mathrm{PPA}$ for the first $5 \mathrm{~d}$, and 4) PPA + EGCG: given $500 \mathrm{mg} / \mathrm{kg} \mathrm{BW}$ sc PPA for $5 \mathrm{~d}$ and $100 \mathrm{mg} / \mathrm{kg}$ EGCG by gavage for the $35 \mathrm{~d}$. The Morris Water Maze (MWM) intelligence test was applied for the last $5 \mathrm{~d}$ of the study. The study lasted 35 days. In rats induced with PPA, EGCG supplementation decreased neurodegeneration by increasing NGF, BDNF, TrkB, and CaMKII- $\alpha$ levels and decreasing CREB levels $(\mathrm{p}<0.001)$. The MWM intelligence test revealed that PPA increased the spatial memory acquisition phase (SMAP) levels $(\mathrm{p}<0.001)$ and decreased the average time spent in the target quadrant compared with control $(\mathrm{p}<0.01)$. As a result of administration of EGCG, a polyphenol, the levels of damage markers formed in the brain tissue approached those in the control group and showed an improvement in rats in which an experimental ASD model was created using PPA. The MWM test also revealed that the administration of EGCG decreased SMAP levels and increased the average time spent in the target quadrant.
\end{abstract}

Keywords: EGCG, propionic acid, autism, NGF, BDNF, TrkB

Autism spectrum disorder (ASD) is a neurodevelopmental disorder that causes difficulties with social communication and interaction and is characterized by atypical, limited, and recurrent behavioral characteristics. It is a common psychiatric pathology that usually occurs in children $<3$ years old affects about $1 \%$ of the world population and has a higher rate in males (male/female ratio, $4.3: 1$ ). Approximately two-thirds of individuals with autistic features have a mental disability; however, the etiology of ASD, including the genetic and environmental factors, has not been fully understood (1-4).

Experimental animal models are often used to test the pathological mechanisms of disease and to identify potential treatments that target the affected metabolic pathways. ASD models can help reveal its etiology and test therapeutic agents $(5,6)$. MacFabe et al. (7) and El-Ansary et al. (8) have suggested that brain infusion or propionic acid (PPA) administration to rat pups may induce most of the biochemical properties seen in individuals with $\operatorname{ASD}(5,7,8)$.
Polyphenols have become increasingly important substances for their beneficial effects on various diseases (9). Epigallocatechin gallate (EGCG), polyphenolic catechin, is the main active antioxidant ingredient found in green tea. EGCG has many important biological properties, including scavenging of free radicals and antitumor effects. Previous studies have indicated that EGCGs healing effects on the heart, kidneys, and intestines also induce apoptosis in damaged cells from ischemia-reperfusion. As EGCG can easily cross the blood-brain barrier, it has recently gathered the attention of researchers conducting neurological studies (10, 11). EGCG prevents the death of neuron cells caused by $\beta$-amyloid neurotoxicity. In addition, EGCG therapy modulates amyloid precursor protein cleavage and decreases cerebral amyloidosis in the mice model of Alzheimer's disease (12). EGCG administered to cultured neurons protects cells against oxidative stress by increasing heme oxyge-

\footnotetext{
* Corresponding author: e-mail: oguzhan.ozdemir@batman.edu.tr; oguzhan23@gmail.com
} 
nase 1 expression by activating transcription factor Nrf2 (13).

Protein synthesis can be modulated by factors that regulate transcription rates such as the CAMPresponsive element-binding protein (CREB). Studies have reported that CREB and Brain-Derived Neurotrophic Factor (BDNF) are critical factors involved in memory formation. CREB, a nuclear protein, plays a role in many important neuronal processes such as development, survival, and neuronal plasticity, which are critical for memory formation (14). In addition, Nerve Growth Factor (NGF) and BDNF signaling pathways are characteristic properties of several central- and peripheral nervous-system disorders, such as dementia, depression, amyotrophic lateral sclerosis, multiple sclerosis, stroke, neuropathy, and eating and nociceptive disorders (15).

Studies using an ASD animal model on the possible effects of EGCG dietary supplement on some of the molecular pathways in brain tissue (e.g., CREB, CaMKII- $\alpha$ ) are limited; therefore, the present study investigated possible effects of EGCG on brain tissue NGF, BDNF, Tropomyosin Receptor Kinase B (TrkB), CREB, and Calcium/CalmodulinDependent Protein Kinase II Subunit Alpha (CaMKII- $\alpha$ ) proteins by using Sprague-Dawley rats in which a PPA-induced experimental ASD model was created.

\section{EXPERIMENTAL}

\section{Experimental animals}

Twenty-eight 21-d-old male Sprague-Dawley rats were used to create a PPA-induced ASD model and to demonstrate the effect of EGCG (Teavigo ${ }^{\circledR}$, DSM, Istanbul, Turkey) treatment on the ASD rats. The animals were obtained from Firat University, Elazig, Turkey, and kept under standard conditions of humidity $(55 \pm 5 \%)$, room temperature (22 \pm $2^{\circ} \mathrm{C}$ ), and 12-h light/dark cycles; they had free access to water and food. The approval from Firat University Animal Care and Use Committee (approval \#2019/10-105) was obtained and the study was performed at the university's Faculty of Veterinary Science. All processes were conducted in strict adherence to related animal welfare laws and policies concerning public health.

\section{Experimental design and PPA-EGCG adminis- tration}

The rats were randomly assigned to the following four groups with seven rats in each: 1) control: given a standard diet, 2) EGCG: given $100 \mathrm{mg} / \mathrm{kg}$ EGCG gavage for $35 \mathrm{~d}, 3$ ) PPA (Sigma-Aldrich. St. Louis, MO, USA): given $500 \mathrm{mg} / \mathrm{kg} \mathrm{BW} \mathrm{sc} \mathrm{PPA} \mathrm{for}$ the first $5 \mathrm{~d}$ and a standard diet for $35 \mathrm{~d}$, and 4) PPA + EGCG: given PPA for the first $5 \mathrm{~d}$ and 100 $\mathrm{mg} / \mathrm{kg} / \mathrm{d}$ EGCG gavage for $35 \mathrm{~d}$. The experimental procedures are shown in Figure 1.

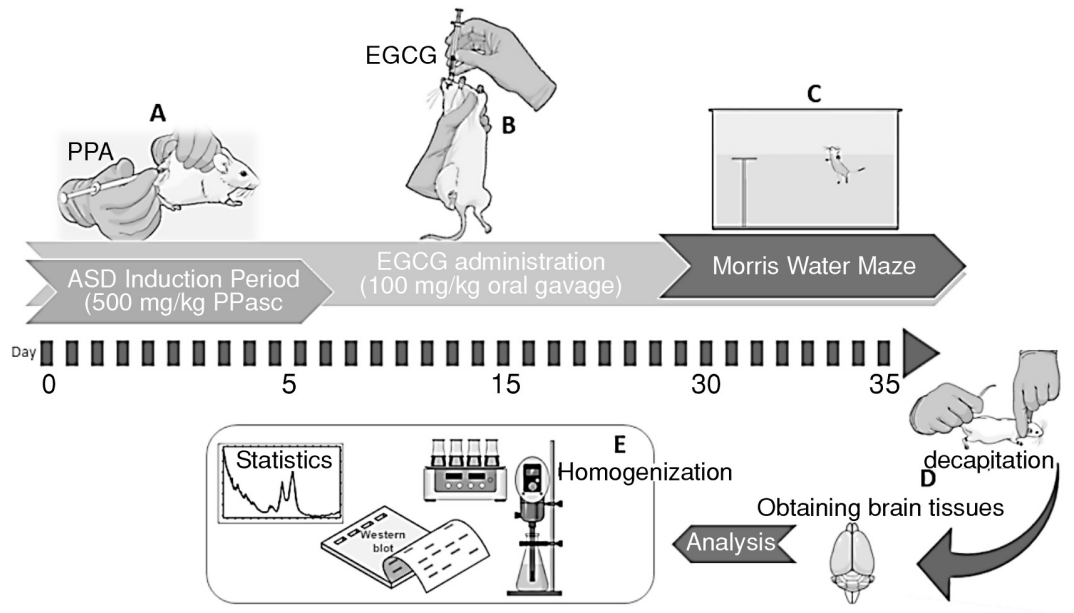

Figure 1. Schematic representation of the experimental design. (A) Autism spectrum disorder (ASD) induction at 0-5 d, (B) epigallocatechin gallate (EGCG) administration (0-35 d), (C) Morris Water Maze (30-35 d), (D) decapitation and extracting brain tissues (day 35), (E) analysis (homogenization, Western blotting, statistical analysis). 

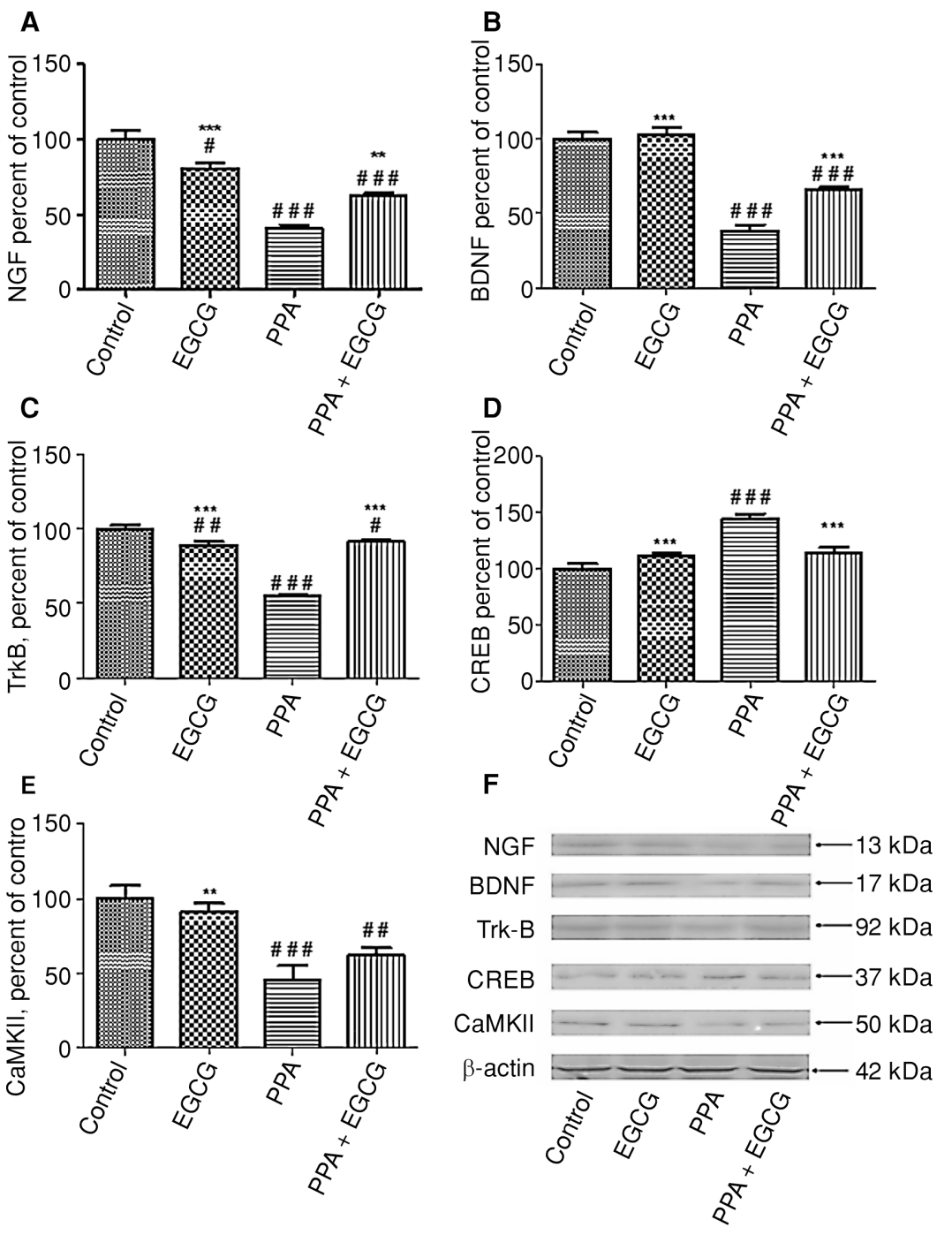

Figure 2. Brain tissue nerve growth factor (NGF), brain-derived neurotrophic factor (BDNF), tropomyosin receptor kinase (TrkB), calcium/calmodulin-dependent protein kinase II subunit alpha (CaMKII- $\alpha$ ) increasing levels and decreasing CAMP-responsive element-binding protein (CREB) expression levels using Western blot strips (above all panel), effect of epigallocatechin gallate (EGCG) on NGF (A), BDNF (B), TrkB (C), CREB (D), and CaMKII- $\alpha$ (E). The intensity of the bands (F) was quantified by densitometric analysis. Protein expression levels in brain tissue of ASD induced using propionic acid (PPA). The intensity of the bands was quantified by densitometric analysis. Data are expressed as a ratio of the normal control value (set to 100\%). The bar represents the standard error of the mean. Blots were repeated at least three times $(n=3)$ and a representative blot is shown. $\beta$-actin was included to ensure equal protein loading. Notes: The mean values on the bars with no common superscript differ significantly at the level of $p<0.05$ using Tukey's multiple comparison test. Data are presented as the mean values \pm standard error of the mean $(\mathrm{SEM}, \mathrm{n}=7)$; ${ }^{*} \mathrm{p}<0.05$ and $* * \mathrm{p}<0.01$ compared with the PPA group; \#p $<0.05$, \# \# < 0.01; and \# \# \# $<0.001$ compared with the control group.

PPA used for the formation of the ASD model (16) and the EGCG doses used for treatment (17) were determined according to previous studies. PPA was dissolved in $0.1 \mathrm{M}$ phosphate-buffered saline and administered subcutaneously at a dose of $500 \mathrm{mg} / \mathrm{kg}(250 \mathrm{mg} / \mathrm{mL}$, $0.26 \mathrm{M}, \mathrm{pH} 7.4$ ) once a day for $5 \mathrm{~d}$. The animals received EGCG by gavage. Subcutaneous saline placebo was administered to the control group in the same dose and duration as that for PPA. Drinking water in equal vol- ume $(1 \mathrm{~mL} / \mathrm{rat})$ was administered to the groups without EGCG by gavage. On day 30, the Morris Water Maze (MWM) memory test was applied. At the end of $35 \mathrm{~d}$, the rats were decapitated within the framework of ethical rules and the target brain tissues were removed.

\section{Western blotting}

Protein levels were measured using Western blotting, as described by Sahin et al. (18). Protein 
concentration was determined using the Lowry method. Sodium dodecyl sulfate-polyacrylamide gel electrophoresis was conducted on 50ug from pooled tissue samples and the samples were subsequently transferred to a nitrocellulose membrane (Schleicher and Schuell Inc., Keene, NH, USA). The primary antibodies against NGF, BDNF, TrkB, CREB, CaMKII- $\alpha$, and $\beta$-actin were purchased from Abcam (Abcam Inc., Cambridge, UK) and antibodies were diluted $(1: 1000)$ in the same buffer containing $0.05 \%$ Tween-20. The nitrocellulose membrane was incubated overnight at $4{ }^{\circ} \mathrm{C}$ with the protein antibody. The blots were washed and incubated with the appropriate horseradish peroxidase-conjugated secondary antibody (goat antimouse immunoglobulin $[\mathrm{Ig}] \mathrm{G})$ at a dilution of $1: 5000$ (Abcam). The procedures were repeated at least four times to confirm data reproducibility. Densitometry was conducted to evaluate protein levels using Image J (National Institutes of Health, Bethesda, MD, USA), corrected with values determined on $\beta$ actin blots, and reported as relative values compared with those in the control group.

\section{Morris Water Maze test}

A MWM tank measuring $150 \times 60 \mathrm{~cm}$ was used to monitor the spatial learning and memory of the rats. To make the 11 - by $11-\mathrm{cm}$ platform invisible, it was painted black and made impossible to distinguish inside of the tank. The tank was filled with water and the temperature of the water was kept at $23^{\circ} \mathrm{C}$ using an automatic heater. A computerized video surveillance system was used to observe the rats in the pool attempting to find the hidden platform according to the extra maze hints. Two different strategies were applied to the rats for place learning. During each trial in the allothetic paradigm, the rats used environmental cues as spatial reference points; in the idiotic paradigm, these cues were blocked by nontransparent screens placed around the pool (19). During place learning, the rats were tested for four consecutive days (allothetic paradigm). The experiment ended when a rat found the platform or when $60 \mathrm{sec}$ had passed. All rat movements (swimming speed, distance and trajectory, and escape delay) were recorded using video surveillance. After the place-learning experiment, the rats were subject-
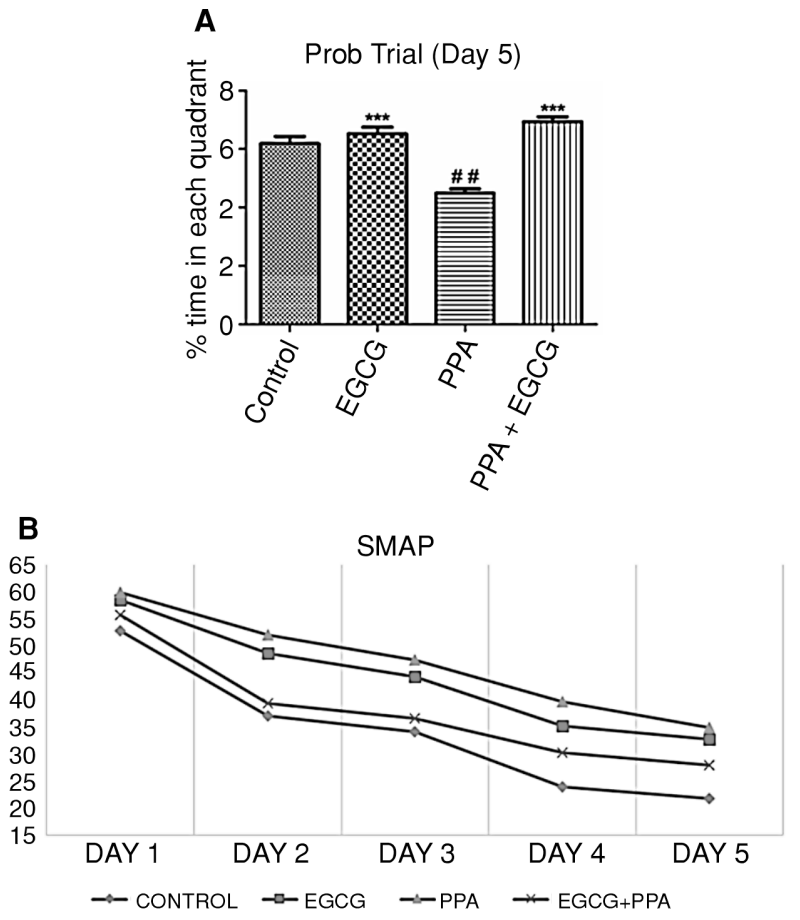

Figure 3. Morris Water Maze test. (A) Probability trial (day 5), (B) memory acquisition phase (days 1-5). Notes: The mean values on the bars with no common superscript differ significantly at the level of $\mathrm{p}<0.05$ using Tukey's multiple comparison test. Data are presented as the mean values \pm standard error of the mean (SEM, $\mathrm{n}=7$ ); *** $\mathrm{p}<0.001$ compared with the PPA group; and \# \#p $<0.01$ compared with the control group. 
ed for 5 consecutive days to the MWM spatial memory acquisition phase (SMAP) (20). In the probe trial, which measures the strength of the acquired chosen location, the rats swam in the pool for $60 \mathrm{sec}$ without a platform included in the water. In the platform trial, the distance and percentage of times spent in an imaginary 40-cm circle around the platform were designated as the "ring 40 ".

\section{Statistical analyses}

Data were analyzed using the Statistical Package for the Social Sciences (SPSS) v. 21 (IBM Corp., Armonk, NY, USA). Protein levels and MWM were measured using one-way analysis of variance (ANOVA). Tukey's multiple comparison test was used to detect changes between the groups. Statistical significance was set at $\mathrm{p}<0.05$.

\section{RESULTS}

\section{Western blotting}

The brain tissue was analyzed for NGF, BDNF, TrkB, CREB, and CaMKII- $\alpha$ protein levels using Western blotting. The levels of brain tissue transcription factors of EGCG supplementation in autism-induced rats after PPA application are shown in Figure 2. NGF, BDNF, TrkB, and CaMKII- $\alpha$ levels decreased and CREB levels increased in the PPA group compared to those in the control group $(\mathrm{p}<$ 0.001). The NGF, BDNF, TrkB, and CaMKII- $\alpha$ levels significantly increased and CREB level significantly decreased $(p<0.001)$ in the PPA + EGCG group compared to those in the PPA group. Transcription factors in the PPA + EGCG group approached those of the control group because of the protein-level changes. Compared to the control group, no significant changes were found in BDNF, CREB, or CaMKII- $\alpha$ levels $(p>0.05)$ and in NGF $(p<0.05)$; whereas, TrkB significantly decreased in the rats that received only EGCG $(\mathrm{p}<0.01)$.

\section{Morris Water Maze}

The MWM test results are shown in Figure 3. Compared to that in the control group, the time spent in the target quadrant decreased by $23.55 \%$ ( $\mathrm{p}<$ 0.01 ) and the SMAP performance values increased by $37.92 \%$ in the PPA group ( $<<0.001)$. In the PPA + EGCG group, the time spent in the target quadrant increased by an average of $45.01 \%(\mathrm{p}<0.001)$, and the mean percentage time spent in SMAP decreased by $18.82 \%(p<0.001)$ compared to those in the PPA group. In the rats treated with EGCG, the average time spent in the target quadrant compared to that of the control group $(p>0.05)$ was not affected; how- ever, the SMAP performance values were increased by $26.49 \%(\mathrm{p}<0.001)$.

\section{DISCUSSION}

In the present study, in the rats given EGCG, a polyphenol, the levels of damage markers formed in the brain tissue approached those of the control group and were improved in the rat experimental ASD model that was created using PPA. The MWM test also revealed that the administration of EGCG decreased SMAP levels and increased the average time spent in the target quadrant.

ASD is a life-long neurodevelopmental disease characterized by disorders in social communication, interactions, recurrent behaviors, interests, and activities. There are no diagnostic tests or treatments for ASD, and Nicolini and Fahnestock (21) have indicated that its etiology contains a mixture of heterogeneous factors. Previous studies have found that neurodegenerative damage is observed in rats after administrating agents such as PPA and valproic acid (VPA) and that these rats have shown ASD symptoms $(16,22)$. Mirza and Sharma (22) have reported that behavioral and biochemical symptoms associated with ASD decrease as a result of treatment with fenofibrate in VPA-induced ASD-induced Wistar rats.

It has been reported that polyphenols have therapeutic values in neurodegenerative, hypertension, cardiovascular, cancer, diabetes, dyslipidemia, allergy, and immune system diseases (13). The action mechanism of polyphenols depends mainly on their antioxidant potential and ability to balance oxidative stresses (23). In addition, many polyphenols are known to interfere with other key cell-signaling pathways, oxidative stress, inflammation, and cell proliferation, such as activator protein 1, nuclear factor kappa B, and the signal transducer and activator of transcription $1(24,25)$. Moreover, dietary polyphenols are versatile compounds and ideal candidates for ASD treatment because they target a large number of molecules. Polyphenols also reach higher concentrations in the human body when absorbed from the intestine after oral administration (24). It has been reported that resveratrol treatment, a polyphenol supplement, reduces symptoms of ASD in the rat model created by VPA prenatal induction (26).

In many treatment areas, EGCG, an important polyphenol component of green tea, has been administered because of its useful bioactivity and easy accessibility $(27,28)$. EGCG appears to have many beneficial effects on the brain, including as a 
powerful antioxidant that functions to prevent oxidative damage in healthy cells. EGCG alleviates ischemia (29), cisplatin-induced cerebral inflammation (30), and Alzheimer's (31) and improves cognitive functions (32). Moreover, more than 90 clinical trials tested the therapeutic potential of EGCG on cancer, heart disease, diabetes, and obesity, and other diseases such as Fragile $\mathrm{X}$ and Down syndromes $(33,34)$. EGCG can act as a chain-breaking antioxidant by inhibiting lipid peroxidation, which represents a potential source of harmful reactive oxygen species in degenerative diseases. It can also affect a wide range of improvement/differentiation signaling transduction pathways, including ERK and PI3K/AKT $(35,36)$. Moreover, EGCG is a more important compound for neurofeedback than other polyphenols because of its ability to easily cross the blood-brain barrier (10). Several studies have reported that EGCG slows the brain aging process and reduces $\beta$-amyloid levels by regulating the amyloidogenic $\alpha$-secretase proteolytic pathway $(37,38)$. In their study on mice, Bao et al. (37) have suggested that EGCG dietary supplement is a safe and effective preservative against Alzheimer's disease. In an in vivo/in vitro study using transgenic mice with synaptic damage caused by CDKL5, Trovňa et al. (36) have reported that EGCG dietary supplement may have a positive effect on neurological defects and cognitive activities associated with CDKL5. In another in vitro/in vivo study, Zhong et al. (39) have found that EGCG administration induced the activation of canonical NOD-, LRRand pyrin domain-containing protein 3 (NLRP3) and noncanonical caspase-11 resulting from the TLR4/NF- $\kappa$ B pathway when it inhibits NLRP3. Zhong et al. (39) have also found that EGCG reduces microglial inflammation and neurotoxicity. EGCG supplementation decreased neurodegeneration by increasing the NGF, BDNF, TrkB, and CaMKII- $\alpha$ levels and decreasing the CREB levels in an ASD-induced rat model using PPA. The results of the MWM intelligence test supported these findings because the SMAP levels decreased and the average time spent in the target quadrant increased. A better understanding of the neuroprotective effects and simultaneous modulation of the signaling pathways of EGCG and other polyphenols is important for determining a treatment for neurodegenerative diseases such as ASD and Alzheimer's.

\section{Acknowledgments}

I extend my gratitude to Prof. Kazim Sahin and his work team for their help in the experiments.

\section{Conflicts of interest}

The author declares no conflict of interest.

\section{REFERENCES}

1. Pascolini G., Majore S., Valiante M., Bottillo I., Laino L., et al.: Psychiatr. Genet. 29, 86 (2019).

2. James B.J., Gales M.A., Gales B.J.: Ann. Pharmacother. 53, 537 (2019).

3. Lassalle A., Zürcher N.R., Porro C.A., Benuzzi F., Hippolyte L., et al.: Soc. Neurosci. 14, 359 (2019).

4. Castro K., Perry I.S., Ferreira G.P., Marchezan J., Becker M., Riesgo R.: J. Autism Dev. Disord. 49, 2536 (2019),

5. Alfawaz H., Al-Onazi M., Bukhari S.I., Binobead M., Othman N., et al.: J. Mol. Neurosci. 66, 403 (2018).

6. Mepham J.R., Boon F.H., Foley K.A., Cain D.P., MacFabe D.F., et al.: Neurotox. Res. 35, 823 (2019).

7. MacFabe D.F., Cain D.P., Rodriguez-Capote K., Franklin A.E., Hoffman J.E., et al.: Behav. Brain. Res. 176, 149 (2007).

8. El-Ansary A.K., Bacha A.B., Kotb M.J.: J. Neuroinflammation 9, 74 (2012).

9. Karatas A., Dagli A.F., Orhan C., Gencoglu H., Ozgen M., et al.: Biotechnol. Appl. Biochem. 67, 317 (2020).

10. Zhou W., Chen L., Hu X., Cao S., Yang J.: Neuroreport. 30, 60 (2019).

11. Kilic U., Sahin K., Tuzcu M., Basak N., Orhan C., et al.: Front. Nutr. 28, 1 (2015).

12. Sharman M.J., Gyengesi E., Liang H., Chatterjee P., Karl T., et al.: Neurobiol. Dis. 124, 505 (2019).

13. Moosavi F., Hosseini R., Saso L., Firuzi O.: Drug. Des. Devel. Ther. 21, 23 (2015).

14. Khalaj-Kondori M., Sadeghi F., Hosseinpourfeizi M.A., Shaikhzadeh-Hesari F., Nakhlband A., et al.: Turk J. Med. Sci. 46,1573 (2016).

15. Chaldakov G.N., Tonchev A.B., Aloe L.: Riv. Psichiatr. 44,79 (2009).

16. Choi J., Lee S., Won J., Jin Y., Hong Y., et al.: PLos One 13, e0192925 (2018).

17. Sahin K., Tuzcu M., Gencoglu H., Dogukan A, Timurkan M., et al.: Life Sci. 87, 240 (2010).

18. Sahin K., Yabas M., Orhan C., Tuzcu M., Sahin T.K., et al.: Expert Opin. Ther. Targets 2, 1 (2020).

19. Elibol-Can B., Dursun I., Telkes I., Kilic E., Canan S., et al.: Dev. Neurobiol. 74, 498 (2014). 
20. Sahin K., Tuzcu M., Orhan C., Agca C.A., Sahin N., et al.: Biol. Trace Elem. Res. 143, 1018 (2011).

21. Nicolini C., Fahnestock M.: Exp. Neurol. 299, 217 (2018).

22. Mirza R., Sharma B.: Brain. Res. Bull. 147, 36 (2019).

23. Lapi D., Stornaiuolo M., Sabatino L., Sommella E., Tenore G., et al: Front. Cell. Neurosci. 14, 3 (2020).

24. Serra D., Almeida L.M., Dinis T.C.P.: Neurosci. Biobehav. Rev. 102, 290 (2019).

25. Tewari S., Dubey K.K., Singhal R.S.: J. Food Sci. Technol. 55, 1525 (2018).

26. Barone R., Rizzo R., Tabbě G., Malaguarnera M., Frye R.E., et al.: Int. J. Mol. Sci. 20, 1878 (2019).

27. Kim S., Kim K., Kim B.S., An Y.H., Lee U.J., et al.: Biomaterials 242, 119905 (2020).

28. Zhang X-N., Liao Y.W.K., Wang X.R., Zhang L., Ahammed G.J., et al.: Plant Physiol. Biochem. 150, 263 (2020).

29. Park D.J., Kang J.B., Koh P.O. J.: Vet. Med. Sci. 82, 639 (2020).

30. Arafa M.H., Atteia H.H.: Neurotox. Res. 37, 380 (2020).
31. Singh N.A., Bhardwaj V., Ravi C., Ramesh N., Mandal A.K.A., et al.: Front. Aging Neurosci. 10, 244 (2018).

32. Wei B.B., Liu M.Y., Zhong X., Yao W.F., Wei M.J.: Acta. Pharmacol. Sin. 40, 1490 (2019).

33. de la Torre R., de Sola S., Hernandez G., Farré M., Pujol J., et al.: Lancet Neurol 15, 801 (2016)

34. Granja A., Frias I., Neves A.R., Pinheiro M., Reis S.: Biomed. Res. Int. 2017, 15 pages (2017)

35. Minnelli C., Galeazzi R., Laudadio E., Amici A., Rusciano D., et al.: Antioxidants 9, 208 (2020).

36. Trovò L., Fuchs C., De Rosa R., Barbiero I., Tramarin M., et al.: Neurobiol. Dis. 138, 104791 (2020).

37. Bao J., Liu W., Zhou H-Y., Gui Y.R., Yang Y.H., et al.: Curr. Med. Sci. 40, 27 (2020).

38. Rezai-Zadeh K., Arendash G.W., Hou H., Fernandez F., Jensen M., et al.: Brain Res. 1214, 177 (2008).

39. Zhong X., Liu M., Yao W., Du K., He M., et al.: Mol. Nutr. Food Res. 63, e1801230 (2019).

C 2020 by Polish Pharmaceutical Society. This is an access article under the CC BY NC license (http://creativecommons.org/licenses/by-nc/4.0/). 